\title{
A Magnetic Spectrometer Analysis of Ultra High Energy Cosmic Ray Arrival Directions
}

\section{Foteini Oikonomou*}

Physics Department, The Pennsylvania State University, University Park, PA 16802, USA

E-mail: oikonomouepsu.edu

\section{Miguel Mostafa}

Physics Department, The Pennsylvania State University, University Park, PA 16802, USA

Email: miguelapsu. edu

\begin{abstract}
We present a method of identifying bright sources of ultra-high energy cosmic rays (UHECRs) embedded in a diffuse background flux by studying UHECR arrival directions. The technique is applicable to sources that produce several observed events, which exhibit energy ordering in their arrival directions due to coherent deflections caused by the regular component of the Galactic magnetic field. We quantify the efficiency of the method with numerical simulations which take into account the possible uncorrelated values of the extragalactic magnetic fields that the UHECRs might encounter, possible turbulent magnetic fields in our own Galaxy, and in particular the non-uniform exposure of UHECR observatories. In the absence of energy ordering in excess of background expectations we place an upper limit on the flux fraction contributed by the brightest UHECR source, which can constrain models of the origin of UHECRs and their composition.
\end{abstract}

35th International Cosmic Ray Conference - ICRC2017

10-20 July, 2017

Bexco, Busan, Korea

${ }^{*}$ Speaker. 


\section{Introduction}

The sources of the highest energy cosmic rays remain unknown despite decades of experimental and theoretical efforts. At present, the Pierre Auger Observatory (hereafter Auger) [1] and the Telescope Array (TA) [2,3] detect the extensive air showers produced when UHECRs enter the Earth's atmosphere in the South and North hemisphere respectively. Together the two experiments observe the full sky.

The sources of observed UHECRs with energies exceeding $\sim 50 \mathrm{EeV}\left(1 \mathrm{EeV}=10^{18} \mathrm{eV}\right)$ must lie within a few hundred Mpc, as above this energy UHECRs lose energy rapidly through interactions with the Cosmic Microwave Background (CMB), primarily via the GZK process [4, 5]. If UHECRs are protons, their trajectories are expected to be deviated by extragalactic magnetic fields by a few degrees at energies exceeding $\sim$ few $\times 10 \mathrm{EeV}$. Around $E \sim 4 \mathrm{EeV}$ the cosmicray spectrum seems to be dominated by protons. Above this energy (known at the "ankle" of the cosmic-ray spectrum) the composition gradually becomes heavier (interpreting the data using standard hadronic interaction models) [6, 7]. The chemical composition of UHECRs at, and above $50 \mathrm{EeV}$ is not well understood, due to low event detection statistics.

If UHECR trajectories are not significantly deviated by extragalactic and Galactic magnetic fields, a correlation is expected between their arrival directions and their sources. Searches for such a correlation to date have not yielded a clear signal $[8,9]$. If the trend to a gradually heavier UHECR composition seen at lower energies continues to beyond the GZK cutoff, it would explain the absence of a strong correlation.

The strength and configuration or Galactic and extragalactic magnetic fields is highly uncertain, contributing to the inability to make conclusions about the origin of UHECRs. The strongest upper bounds on the extragalactic magnetic field strength to date, come from the measurement (or absence of) polarisation in the radio emission of distant quasars [10]. They constrain the average strength of extragalactic magnetic fields to be $B \lambda_{B}^{1 / 2} \lesssim 1.7 \times 10^{-9} \mathrm{GMpc}^{1 / 2}$, with $\lambda_{B}$ the coherence length of the field. The deflection of UHECRs in extragalactic magnetic fields is likely subdominant with respect to the deflections caused by the Galactic Magnetic Field. The most complete attempts to date to model the Galactic magnetic field, were developed based on the largest available datasets of Faraday rotation measurements, and polarised synchrotron emission [11, 12], but uncertainties remain non-negligible.

The possibility to detect several UHECRs from a single source is a promising way of reconstructing the source position as well as the integral strength of the magnetic field perpendicular to the UHECR trajectory between the source and Earth. Interestingly, both Auger and TA have both reported hints for an excess of arrival directions at intermediate scales above $50 \mathrm{EeV}[8,13]$. Whether these observations are due to single powerful UHECR sources, an excess of UHECR sources in these directions, or statistical fluctuations remains to be seen with further data, or novel analyses.

A number of studies have been conducted to date on the topic of multiplets $[14,15,16,17$, 18]. In this paper we revisit the possibility of detecting multiplets of UHECRs originating in a common source, by studying the signal that would be imprinted in the UHECR arrival directions with simulations of UHECRs from extragalactic sources. We improve on the search for multiplets with respect to earlier work on the topic in a number of ways. Firstly, we quantify the sensitivity 
of our algorithm by use of simulations that rely on the most recent parameterisations of Galactic magnetic fields $[11,12]$. In addition, this is the first study on multiplets to consider the possible effect of the extragalactic, as well as regular, and turbulent Galactic magnetic field components. Importantly, we consider in this work for the first time, in a search for UHECR energy ordering, the effect of a non-uniform, partial sky exposure, that reflects the non-uniform exposure of true UHECR detectors, to the sensitivity of such UHECR detectors to any true multiplets.

\section{Formalism}

Two or more UHECRs that originate in a common source, that get deviated by the same, large scale magnetic field, should experience a deflection inversely proportional to their individual energies, if they have the same charge. We refer to this as the "magnetic spectrometer effect".

Formally, a pair of events $(i, j)$ from a common source, with energies $E_{i}, E_{j}$, will experience deflections of unknown strength $\theta_{i}, \theta_{j}$ with respect to their source. Let us assume that $E_{i}>E_{j}$ in the present example. If these events have been deviated by a common, regular magnetic field, each will experience a deflection $\theta$ (formally $\theta$ is the angle between the source and the arrival direction of the UHECR), given by:

$$
\sin \theta=\left(\frac{c e D|\mathbf{B}|}{2}\right) \frac{Z}{E}
$$

where $D$ is the distance to the source, and B is the strength of the uniform magnetic field, perpendicular to the motion of the UHECR.

If the two UHECRs have the same electric charge, the following relation applies:

$$
\frac{\sin \theta_{i}}{\sin \theta_{j}}=\frac{E_{j}}{E_{i}}
$$

Eq. 2.2 gives

$$
\theta_{j}=\sin ^{-1}\left(\frac{E_{i}}{E_{j}} \sin \theta_{i}\right) .
$$

As the angle between events $i$, and $j, \Delta \theta$ is known, one can solve eq. 2.3 for $\theta_{i}$ :

$$
\begin{gathered}
\Delta \theta=\theta_{j}-\theta_{i}=\sin ^{-1}\left(\frac{E_{i}}{E_{j}} \sin \theta_{i}\right)-\theta_{i} \\
\therefore \theta_{i}=\sin ^{-1}\left(\frac{\sin \Delta \theta}{\frac{E_{i}}{E_{j}}-\cos \Delta \theta}\right) .
\end{gathered}
$$

We use equations 2.4,2.5 to define the nominal direction of the source of any pair of events.

For each pair of events that survive our criteria (see later) in a set of UHECRs we calculate the nominal source position. To take into account energy and directional uncertainties we repeat the procedure 1000 times for each event pair, resampling each time the energy and arrival direction of each event within the detector resolution. The procedure described results in a large number of nominal source positions being calculated. Once the source positions for every pair of events have been calculated the resulting skymap is smoothed with a top hat filter. Each smoothed map results in one or more high density nominal source directions. Figure 1 shows an example of such 


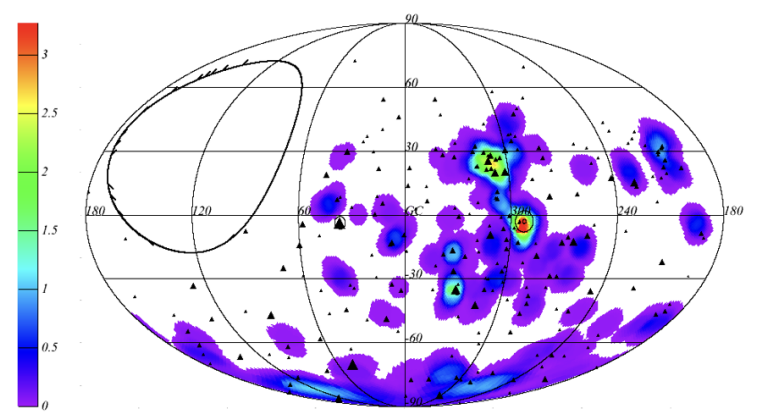

Figure 1: Skymap of simulated arrival directions from four powerful UHECR sources, producing several observed events each, embedded in an isotropic background that follows the energy, and spatial distribution of events as might have been seen at the Pierre Auger Observatory, in Galactic coordinates. The color-scale gives the normalised density of reconstructed source directions. The size of the triangles is proportional to the events' energy.

a skymap, for a simulated set of events, as might have been observed by Auger. The essence of our approach is to determine whether the brightest point identified in the smoothed skymap of nominal source directions for a given dataset, is compatible with background expectations, or whether there is evidence of energy ordering in the data, beyond expected background fluctuations. We address this question by creating datasets of isotropic arrival directions (after accounting for the exposure of the UHECR detector) in which we embed simulated signal events from a powerful UHECR source, as detailed next.

To correct for the non-uniform exposure of Auger (and all UHECR detectors to date) we assign weights to the reconstructed source directions of all selected pairs. The weight of each pair's nominal source direction is inversely proportional to the time-averaged acceptance of the Auger surface detector at the declination of the lower energy event in the pair (the exposure of Auger is uniform in right ascension, see e.g. [19]). As we show in section 4 this significantly improves the performance of the method, by ensuring that excesses of events are weighted according to how likely they are to occur at a given location in the sky, which varies as a function of declination.

\section{Simulations}

We simulate the signal hypothesis, by considering extragalactic sources at different distances that produce two or more observable events. Throughout, we consider UHECRs with measured energy $E \geq 52 \mathrm{EeV}$. In what follows we concentrate on UHECR protons. In forthcoming work we will extend the analysis to heavier compositions. We consider two extreme scenarios for the energy spectrum of the events produced by a given source; (a) a source with an arriving spectrum that follows an $\mathrm{d} N / \mathrm{d} E \propto E^{-\alpha}$ power-law with index $\alpha=2.0$, and (b) a source with a steep power-law spectrum with index $\alpha=4.2$, motivated by the measured slope of the diffuse spectrum observed at Auger beyond the spectral break at $\sim 43 \mathrm{EeV}$. A source with a soft spectrum as in the latter scenario is intuitively expected to be more easily detectable with the spectrometer algorithm.

The displacement of UHECRs during their extragalactic propagation, is likely much larger than individual magnetised scattering centres (coherent over length scale $\lambda$ ). As shown in $[20,21]$ the arrival directions of different UHECRs from the same source are essentially independent, while 


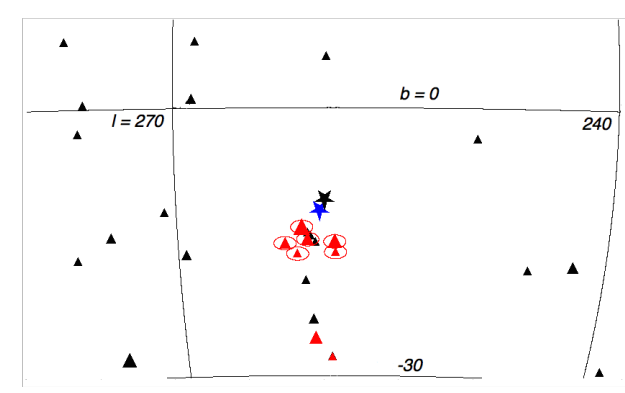

Figure 2: Example of the performance of the spectrometer algorithm, applied to a simulated powerful UHECR source (black star) embedded in an isotropic background. UHECR arrival directions are shown by triangles, with size proportional to energy. UHECRs that the spectrometer algorithm associated with an event cluster are shown with red triangles. Circled triangles indicate the UHECRs that truly originated in the simulated source. The blue star shows the position of the source, as reconstructed by the spectrometer algorithm.

the image of a bright source, prior to Galactic magnetic field (GMF) deflections is essentially Gaussian. In what follows, we model the deflections of UHECRs as a Gaussian with $P(E, \theta)=$ $\exp \left(-\theta_{\text {sim }} / \theta_{s}^{2}\right)$, where $\theta_{s}^{2}=q_{e} *\left(B^{2} \lambda / 9\right)^{1 / 2} / E$, with $q_{e}$ the electron charge, $B$ the average strength of the magnetic field in the line of sight [20]. In this study we fix $B=1 \mathrm{nG}$, and $\lambda=1 \mathrm{Mpc}$. To model the likely effect of the regular component of the GMF we consider the model of [11] (hereafter JF12), and that of [12] (hereafter PT11). For the random GMF we consider several realisations of a turbulent random field whose r.m.s value is that of the JF12 random field model [11], each characterised by a Kolmogorov power spectrum with different maximum coherence lengths. We use the numerical code CRT [22] to simulate the trajectories of UHECRs in the GMF.

We generate background UHECRs according to the exposure of the Pierre Auger observatory. The maximum zenith angle observable by Auger is set to $\theta_{\max }=80^{\circ}$. The energies of background events are generated according to the energy spectrum measured by Auger.

To reduce accidental pairs we impose several constraints to the selected pairs. We restrict the analysis to $\theta_{j}<15^{\circ}$ motivated by the observation that in the models of [11, 12], $\geq 95 \%$ of UHECR protons with $E \geq 50 \mathrm{EeV}$ suffer deflections smaller than $15^{\circ}$. We only consider pairs with $E_{i} / E_{j}>1.02$.

\section{Results}

We apply the algorithm to simulated datasets with background only, or signal and background hypothesis, in order to assess the power of the spectrometer method to successfully identify bright UHECR sources over background. We simulate 1000 isotropically distributed sources over the full sky, with observable flux that varies between few percent and few $\times 10 \%$ of the total UHECR flux above $52 \mathrm{EeV}$. We simulate datasets of 230 events, inspired by the fact that the arrival directions of the 232 most energetic UHECRs have been published by the Auger Collaboration so far. We find that for the presently available number of events a marginally detectable source produces $\sim 5 \%$ of the total UHECR flux. 

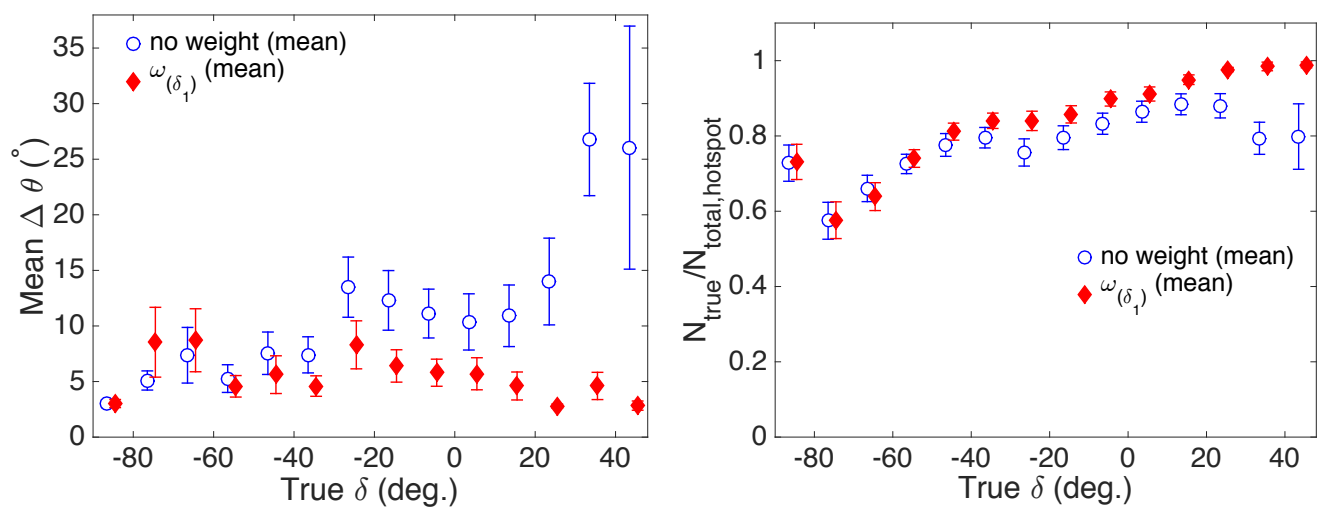

Figure 3: Left: The mean angular error with which the source position is reconstructed in simulations of 1000 sources that produce 5\% of the Auger flux beyond $52 \mathrm{EeV}$ as a function of declination. Right: The fraction of true source events, $N_{\text {true }}$ over the total number of events identified as source events $N_{\text {total,hotspot }}$, as a function of declination.

The left panel of figure 3 shows the mean angular error with which the source position is reconstructed in simulations of 1000 sources that produce 5\% of the Auger flux beyond $52 \mathrm{EeV}$ as a function of declination, for sources with a power-law spectrum with index $\alpha=4.2$. We find that the mean error is less than $5^{\circ}$ for most source directions, and $10^{\circ}$ over the entire Auger field of view. This figure also illustrates the importance of accounting for the detector's non-uniform exposure. If left untreated, large errors occur at northernly declinations, close to the edge of the field of view of Auger.

The right panel quantifies the purity of the "hotspots" detected by the spectrometer algorithm, by showing the fraction of true source events, $N_{\text {true }}$ over the total number of events identified as source events $N_{\text {total,hotspot, }}$ as a function of source declination. The mean of this quantity ranges from 0.6 at the centre of the Auger field of view, where background clusters of events are more likely to $\sim 1$ at northernly declinations, where the background is strongly suppressed, especially after applying weights to account for the exposure of the detector.

Figure 4 shows the distribution of the number of events associated with the brightest source in 1000 realisations of sources of 12 events with a spectrum with $\alpha=2.0$ and $\alpha=4.2$. The important point here is that detected events clusters that contain 8 or more events are inconsistent with a background fluctuation.

\section{Discussion/Conclusions}

We have presented a method to search for clusters of UHECRs, plausibly attributable to astrophysical sources, or concentrations of sources, by searching for energy ordering in the arrival directions of UHECRs, in excess of background expectations.

We have shown that the spectrometer algorithm presented herein is able to succesfully distinguish sources which have produced $\gtrsim 5 \%$ of events detected by Auger with energy above $52 \mathrm{EeV}$, and to point to the source location with uncertainty $\lesssim 5^{\circ}$ for most source directions, and uncertainty $\lesssim 10^{\circ}$ over the entire Auger field of view. 


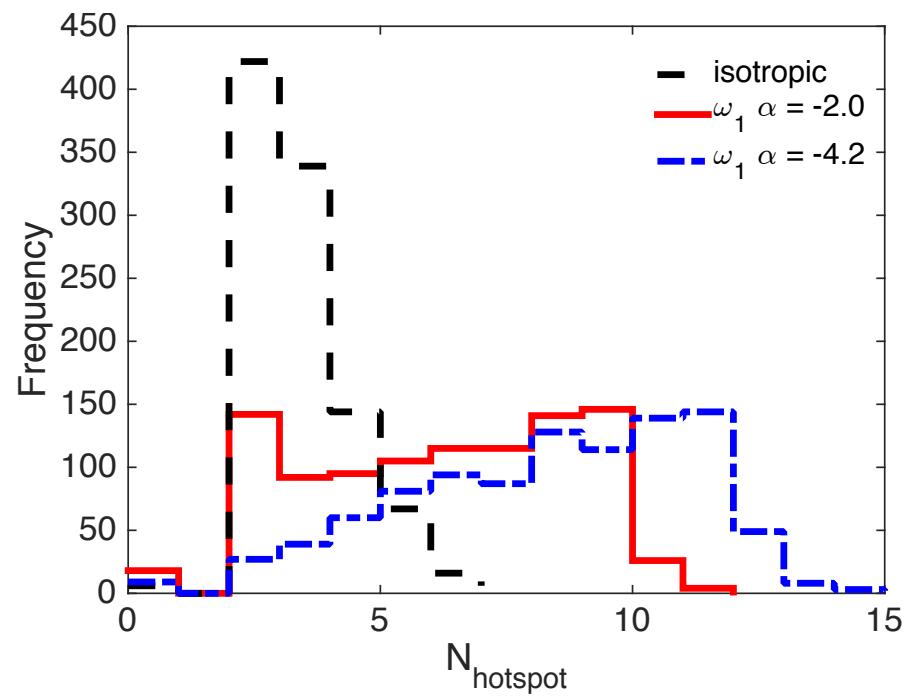

Figure 4: The distribution of numbers of events associated with the brightest source in 1000 background only realisations of 232 Auger events above $50 \mathrm{EeV}$, and in 1000 realisations of sources of 12 events with a spectrum with $\alpha=2.0$ and $\alpha=4.2$.

We have demonstrated the importance of accounting for the non-uniform exposure of UHECR observatories, by showing, in figure 3, the significant improvement in the purity, and mean error on the reconstructed source location, when this important effect is taken into account, by means of applying weights to the reconstructed source positions.

We envisage the application of the method presented here to publicly available Auger and TA datasets.

\section{References}

[1] Pierre Auger Collaboration collaboration, J. Abraham, M. Aglietta, I. C. Aguirre, M. Albrow, D. Allard, I. Allekotte et al., Properties and performance of the prototype instrument for the Pierre Auger Observatory, NIMPA 523 (May, 2004) 50-95.

[2] Telescope Array collaboration, T. Abu-Zayyad, R. Aida, M. Allen et al., The surface detector array of the telescope array experiment, Nuclear Instruments and Methods in Physics Research Section A: Accelerators, Spectrometers, Detectors and Associated Equipment 689 (2012) 87 - 97.

[3] Telescope ARray collaboration, H. Tokuno, Y. Tameda, M. Takeda et al., New air fluorescence detectors employed in the telescope array experiment, Nuclear Instruments and Methods in Physics Research Section A: Accelerators, Spectrometers, Detectors and Associated Equipment 676 (2012) 54 -65 .

[4] K. Greisen, End to the cosmic-ray spectrum?, Phys. Rev. Lett. 16 (1966) 748-750.

[5] G. Zatsepin and V. Kuzmin, Upper limit of the spectrum of cosmic rays, J. Exp. Theor. Phys. Lett. 4 (1966) 78-80.

[6] Pierre Auger collaboration, A. Aab et al., Depth of maximum of air-shower profiles at the Pierre

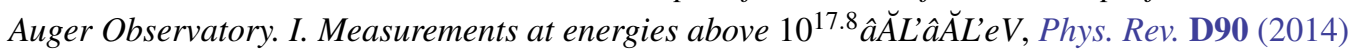
122005, [1409.4809]. 
[7] Pierre Auger, Telescope Array collaboration, R. Abbasi et al., Report of the Working Group on the Composition of Ultra High Energy Cosmic Rays, JPS Conf. Proc. 9 (2016) 010016, [1503.07540].

[8] Pierre Auger collaboration, A. Aab et al., Searches for Anisotropies in the Arrival Directions of the Highest Energy Cosmic Rays Detected by the Pierre Auger Observatory, Astrophys. J. 804 (2015) 15, [1411.6111].

[9] F. Oikonomou, A. Connolly, F. B. Abdalla, O. Lahav, S. A. Thomas, D. Waters et al., A search for correlation of ultra-high energy cosmic rays with IRAS-PSCz and 2MASS-6dF galaxies, 5 (May, 2013) 15, [1207.4043].

[10] M. S. Pshirkov, P. G. Tinyakov and F. R. Urban, New limits on extragalactic magnetic fields from rotation measures, Phys. Rev. Lett. 116 (May, 2016) 191302.

[11] R. Jansson and G. R. Farrar, A New Model of the Galactic Magnetic Field, 757 (Sept., 2012) 14, [1204.3662].

[12] M. S. Pshirkov, P. G. Tinyakov, P. P. Kronberg and K. J. Newton-McGee, Deriving the Global Structure of the Galactic Magnetic Field from Faraday Rotation Measures of Extragalactic Sources, 738 (Sept., 2011) 192, [1103.0814].

[13] Telescope Array collaboration, R. U. Abbasi et al., Indications of Intermediate-Scale Anisotropy of Cosmic Rays with Energy Greater Than 57 EeV in the Northern Sky Measured with the Surface Detector of the Telescope Array Experiment, 790 (2014) L21, [1404.5890].

[14] G. Golup, D. Harari, S. Mollerach and E. Roulet, Source position reconstruction and constraints on the galactic magnetic field from ultra-high energy cosmic rays, 32 (Dec., 2009) 269-277, [0902.1742].

[15] Pierre Auger Collaboration, P. Abreu, M. Aglietta, E. J. Ahn, I. F. M. Albuquerque, D. Allard et al., Search for signatures of magnetically-induced alignment in the arrival directions measured by the Pierre Auger Observatory, 35 (Jan., 2012) 354-361, [1111.2472].

[16] B. Whelan, A Magnetic Spectrometer Analysis Method for Ultra High Energy Cosmic Ray Data. PhD thesis, University of Adelaide, 2011.

[17] G. Giacinti, X. Derkx and D. V. Semikoz, Search for single sources of ultra high energy cosmic rays on the sky, 3 (Mar., 2010) 22, [0907.1035].

[18] G. Giacinti and D. V. Semikoz, Method to look for imprints of ultrahigh energy nuclei sources, 83 (Apr., 2011) 083002, [1011.6333].

[19] P. Sommers, Cosmic ray anisotropy analysis with a full-sky observatory, Astroparticle Physics 14 (Jan., 2001) 271-286, [astro-ph/0004016].

[20] E. Waxman and J. Miralda-Escudé, Images of Bursting Sources of High-Energy Cosmic Rays: Effects of Magnetic Fields, ApJl 472 (Dec., 1996) L89+, [astro-ph/9607059].

[21] J. Miralda-Escudé and E. Waxman, Signatures of the Origin of High-Energy Cosmic Rays in Cosmological Gamma-Ray Bursts, ApJl 462 (May, 1996) L59+, [astro-ph/9601012].

[22] M. S. Sutherland, B. M. Baughman and J. J. Beatty, CRT: A numerical tool for propagating ultra-high energy cosmic rays through Galactic magnetic field models, Astroparticle Physics 34 (Nov., 2010) 198-204, [1 010 .3172]. 